\title{
Was versteht man eigentlich unter sozialer Kompetenz?
}

Soziale Kompetenz bezeichnet die Gesamtheit aller persönlichen Verhaltensweisen und Fähigkeiten, die es einem Individuum ermöglichen und erleichtern, in der Interaktion sowohl mit einzelnen als auch in der Gruppe, in einen in Art und Weise angemessenen und effektiven zwischenmenschlichen Umgang und Austausch zu treten. Der in diesem Zusammenhang zugrundeliegende Kompetenzbegriff geht über die Dimension des formalen Wissens hinaus, er ergänzt sie vielmehr um die des Könnens. Ansatzpunkt für diese Konkretisierung der Dimensionen sozialer Kompetenz sind die sozialen Anforderungen und Bedingungen in zwischenmenschlichen Interaktionssituationen und nicht allein das Individuum. Soziale Kompetenz ist somit nicht nur in einem Bezug zu dem Individuum zu sehen, sondern auch zu der jeweiligen Situation und ihren kulturell spezifischen Anforderungen. Vor diesem Hintergrund wird deutlich, dass in unterschiedlichen Milieus oder Kulturkreisen ähnliche Situationen unterschiedliche situative Anforderungen haben können, d. h., dass in einem Milieu oder Kulturkreis ein bestimmtes Verhalten als sozial kompetent bewertet werden kann, während es in anderen Milieus als sozial inkompetent gilt. Dementsprechend ist die genaue Festlegung der inhaltlichen Dimensionen der sozialen Kompetenz nicht möglich.

Als psychologischer Begriff war soziale Kompetenz bis Mitte des 20. Jahrhunderts gleichrangig mit dem der Intelligenz ein Beurteilungskriterium, ob eine geistige Behinderung vorlag. Inzwischen hat sich der Intelligenzquotient als Maßstab durchgesetzt, obwohl in letzter Zeit eine Tendenz zu beobachten ist, erneut soziale Fähigkeiten und Können mit einzubeziehen.

Nach Holtz können soziale Kompetenzen in aufsteigenden Schwierigkeitsgraden wie folgt definiert werden:

1. Ausdruck: Der Mensch ist in der Lage, sich mit seinen eigenen Wünschen, Meinungen und Wissen einzubringen und verständlich zu machen.

2. Empfang: Es besteht die Fähigkeit zuzuhören, andere Gruppenmitglieder zu beobachten, Ereignisse und gruppendynamische Prozesse bewusst wahrzunehmen.

3. Offenheit: Der Mensch ist offen für Anregungen, kann Kritik akzeptieren und ist bereit, sich mit anderen auseinanderzusetzen.

4. Kooperation: Eigene Handlungsmöglichkeiten und Verantwortlichkeiten werden einerseits erkannt und andererseits besteht die Fähigkeit, sich auf Handlungen von anderen einzustellen und anzupassen.

5. Gestaltung: Der Mensch ist in der Lage, Beziehungen aufzunehmen und zu gestalten, sich in einer Gruppe zurechtzufinden, situationsadäquat zu kritisieren; in gruppendynamischen Prozessen verhält er sich angemessen.

6. Identifikation: Die Fähigkeit, sich auf andere einzustellen und Konflikte situationsgerecht anzugehen, und ein Bewusstsein über die eigenen Möglichkeiten und Grenzen bestehen.

Voraussetzung für sozial kompetentes Verhalten bei Kindern ist nach Petermann, dass sie einerseits frei von sozialer Angst sind und andererseits über soziale Fertigkeiten verfügen. Grundgedanke ist die Beobachtung, dass von sozialer Angst betroffene Kinder Situationen zu vermeiden versuchen, in denen sie in eine soziale Interaktion mit anderen, ihnen fremden Menschen eintreten müssen. Die Folge ist die Ausbildung und Manifestierung eines Vermeidungsverhaltens. Das Fehlen von sozialer Angst wird von Petermann als notwendige Motivvoraussetzung für die Aufnahme einer als normal definierten Kommunikation verstanden. Dabei bildet ein positives Selbstkonzept die Grundlage für das Nichtvorhandensein von sozialer Angst. Weiter wird die Verfügbarkeit und die Möglichkeit des Rückgriffs auf soziale Fertigkeiten als Handlungsvoraussetzung definiert, die durch die Fähigkeit zur Wahrnehmung und Rollenübernahme entsteht. Soziale Angst und soziale Fähigkeiten stehen in einem reziproken Verhältnis. Trotz des generellen Vorhandenseins sozialer Fähigkeiten kann ihr Einsatz und bewusste Verwendung durch soziale Angst behindert oder vermieden werden. In der Folge können soziale Kompetenzen nicht ausgebaut und weiterentwickelt werden. Durch das wiederholte Erleben von Unsicherheit und Misserfolgen wiederum können Verhaltensauffälligkeiten, insbesondere aggressiver Art, ausgebildet werden. Psychologische Trainingsprogramme zur Verbesserung der sozialen Kompetenz bei bestehender sozialer Angst müssen daher zunächst die vorhandenen Unsicherheiten in sozialen Interaktionssituationen fokussieren und durch eine Verbesserung des Selbstkonzeptes zum Angstabbau beitragen.

In den vergangenen Jahren hat der Begriff der sozialen Kompetenz Einzug in die Arbeitswelt gehalten. Soziale Kompetenz gehört hier zu den wichtigsten »soft skills «, den weichen, im Gegensatz zu formalen Qualifikationen (»hard skills«) nicht objektiv nachprüfbaren, Fähigkeiten der Menschen. Soziale Kompetenz wird demnach als wesentliche Schlüsselqualifikation zur Bewältigung von beruflichen Anforderungen, insbesondere auch in Führungspositionen, verstanden. Universitäten, Fachhochschulen und auch private Weiterbildungsinstitute haben auf diese Entwicklung reagiert und berücksichtigen diese Aspekte bewusst bei der Entwicklung ihrer Curricula, die nun neben fachlichen auch den Erwerb von sozialer Kompetenz und Transferkompetenzen enthalten, bzw. konzipieren gezielte Kurse und Seminare zur Verbesserung der Teamfähigkeit, Kommunikationsfähigkeit und des Einfühlungsvermögens. Gleichwohl ist der grundlegende Erwerb von sozialer Kompetenz im Kindesalter leichter als im Erwachsenenalter. Die Förderung des Erwerbs von sozialer Kompetenz sollte daher frühzeitig ansetzen und durch entsprechende Trainingsprogramme unterstützt werden.

Petra Mund

Dr. Petra Mund ist wissenschaftliche Referentin beim Deutschen Verein für öffentliche und private Fürsorge und Lehrbeauftragte an der Alice Salomon Hochschule Berlin. Quelle: Deutscher Verein für öffentliche und private Fürsorge e. V. (Hg.): Fachlexikon der sozialen Arbeit. 6. Auflage. Nomos Verlagsgesellschaft, Baden-Baden 2007. 1.195 Seiten. 44,- Euro. ISBN 978-3-8329-1825-5. Seite 855 f. 